See discussions, stats, and author profiles for this publication at: https://www.researchgate.net/publication/325349108

\title{
Parathyroidectomy for adults with primary hyperparathyroidism
}

Article in Cochrane database of systematic reviews (Online) · May 2018

DOI: 10.1002/14651858.CD013035

CITATION

1

4 authors:

\section{Joseph M Pappachan}

Lancashire Teaching Hospitals NHS Foundation Trust

67 PUBLICATIONS 1,069 CITATIONS

SEE PROFILE

Ananth K. Viswanath

National Health Service

18 PUBLICATIONS 88 CITATIONS

SEE PROFILE

Some of the authors of this publication are also working on these related projects:

Project Diabetes diagnosis View project

Project INCRETIN AND DIABETES View project
READS

81

Ravinder Sodi

University Hospitals Of Morecambe Bay NHS Foundation Trust \& Lancaster Univer...

37 PUBLICATIONS 219 CITATIONS

SEE PROFILE

Ian Lahart

University of Wolverhampton

60 PUBLICATIONS 351 CITATIONS

SEE PROFILE 


\section{(E) Cochrane Library}

Cochrane Database of Systematic Reviews

\section{Parathyroidectomy for adults with primary hyperparathyroidism (Protocol)}

Pappachan JM, Sodi R, Viswanath AK, Lahart IM

Pappachan JM, Sodi R, Viswanath AK, Lahart IM.

Parathyroidectomy for adults with primary hyperparathyroidism.

Cochrane Database of Systematic Reviews 2018, Issue 5. Art. No.: CD013035.

DOI: 10.1002/14651858.CD013035.

www.cochranelibrary.com 


\section{TABLE OF CONTENTS}

HEADER . . . . . . . . . . . . . . . . . . . . . . . . . . . . . . . . . . . . . . . . 1

ABSTRACT . . . . . . . . . . . . . . . . . . . . . . . . . . . . . . . . . . . . . . 1

BACKGROUND . . . . . . . . . . . . . . . . . . . . . . . . . . . . . . . . . . . . 1

OBJECTIVES . . . . . . . . . . . . . . . . . . . . . . . . . . . . . . . . . . . . . . 4

METHODS . . . . . . . . . . . . . . . . . . . . . . . . . . . . . . . . . . . . . . . 44

ACKNOWLEDGEMENTS . . . . . . . . . . . . . . . . . . . . . . . . . . . . . . . . . . . . . . . .

REFERENCES . . . . . . . . . . . . . . . . . . . . . . . . . . . . . . . . . . . . . . . . 9

APPENDICES . . . . . . . . . . . . . . . . . . . . . . . . . . . . . . . . . . . . . . . . . . . . . . .

CONTRIBUTIONS OF AUTHORS . . . . . . . . . . . . . . . . . . . . . . . . . . . . . . . . . . . . . . . 17

DECLARATIONS OF INTEREST . . . . . . . . . . . . . . . . . . . . . . . . . . . . . . . . . . . . . . .

NOTES . . . . . . . . . . . . . . . . . . . . . . . . . . . . . . . . . . . . . . . . 17 
[Intervention Protocol]

\title{
Parathyroidectomy for adults with primary hyperparathyroidism
}

\author{
Joseph M Pappachan ${ }^{1}$, Ravinder Sodi ${ }^{2}$, Ananth K Viswanath ${ }^{3}$, Ian M Lahart ${ }^{4}$ \\ ${ }^{1}$ Endocrinology, University Hospitals of Morecambe Bay NHS Foundation Trust, Lancaster, UK. ${ }^{2}$ Clinical Sciences, University Hospi- \\ tals of Morecambe Bay NHS Foundation Trust, Lancaster, UK. ${ }^{3}$ Endocrinology, New Cross Hospital, Wolverhampton, UK. ${ }^{4}$ Faculty \\ of Education, Health and Wellbeing, University of Wolverhampton, Walsall, UK
}

Contact address: Joseph M Pappachan, Endocrinology, University Hospitals of Morecambe Bay NHS Foundation Trust, Ashton Road, Lancaster, LA1 4RP, UK. drpappachan@yahoo.co.in, pappachan.joseph@mbht.nhs.uk.

Editorial group: Cochrane Metabolic and Endocrine Disorders Group.

Publication status and date: New, published in Issue 5, 2018.

Citation: Pappachan JM, Sodi R, Viswanath AK, Lahart IM. Parathyroidectomy for adults with primary hyperparathyroidism. Cochrane Database of Systematic Reviews 2018, Issue 5. Art. No.: CD013035. DOI: 10.1002/14651858.CD013035.

Copyright (C) 2018 The Cochrane Collaboration. Published by John Wiley \& Sons, Ltd.

\begin{abstract}
A B S T R A C T
This is a protocol for a Cochrane Review (Intervention). The objectives are as follows:

To assess the effects of parathyroidectomy for adults with PHPT. We will also compare the effects of different types of parathyroidectomy on people with PHPT.
\end{abstract}

\section{B A C K G R O U N D}

\section{Description of the condition}

Primary hyperparathyroidism

(PHPT) is an endocrine disorder that affects approximately $1 \%$ of the adult population, and is the third most common endocrine disorder after diabetes mellitus and hypothyroidism (Best 2017). Incidence of the disease increases in older age groups, with the highest prevalence being about $2 \%$ in postmenopausal women in the USA (Ning 2009). The disease is caused by a benign parathyroid adenoma in approximately $80 \%$ of cases, as a consequence of multigland disorder (multiple adenomas or multigland hyperplasia) in $15 \%$ to $20 \%$ of cases, or, in rare cases, a parathyroid carcinoma (Bilezikian 2017). Usually PHPT is associated with hypercalcaemia, elevated or high normal parathyroid hormone (PTH) levels, hypercalciuria, renal stones and bone demineralisation that results in osteopenia or osteoporosis. Although most people with the disease remain asymptomatic prior to the diagnosis with only mild disease, some people may present with symptomatic hypercalcaemia, dehydration leading to acute kidney injury, fragility fractures from osteoporosis, hypertension or chronic kidney disease that prompts clinicians to investigate for PHPT. Elevated PTH levels result in increased bone turnover and skeletal demineralisation. Increased bone turnover leads on to gradual loss of cortical bone mineral density with relative sparing of trabecular bones, and results in predominant osteopenia or osteoporosis of the lumbar spine, neck of femur and forearm bones (Khan 2017; Silverberg 2014). This bone mineral density loss can lead to bone fractures of these sites even with minimal trauma (fragility fractures) which increases morbidity and premature mortality risk, especially in older people with the disease. Alterations in calcium metabolism and bone turnover result in hypercalcaemia, hypercalciuria and nephrolithiasis in a significant proportion of people 
with PHPT. Recent data suggest a prevalence of renal stone disease in $10 \%$ to $20 \%$ of individuals with PHPT (Cipriani 2015; Verdelli 2017). Furthermore, hypertension is found in $40 \%$ to $65 \%$ of people with PHPT, which increases the risk of cardiovascular morbidity and premature mortality (Pepe 2017). PHPT has also been associated with psychoneurological and cognitive dysfunction (Kreidieh 2013; Trombetti 2016). In a proportion of individuals with PHPT serum calcium levels may be consistently in the normal laboratory range and these cases constitute normocalcaemic PHPT. The metabolic and end organ complications in these individuals may be similar to those with classical PHPT (Yener 2016). Overall, PHPT is associated with excess morbidity risk and higher risk of premature mortality.

\section{Description of the intervention}

Surgical removal of abnormal parathyroid tissue (parathyroidectomy) is the optimal treatment for people with proven PHPT. Operative removal of the diseased parathyroid gland(s) removes the source of excess PTH production, and thereby cures the disease in most individuals (Kreidieh 2013). International consensus recommends that all people with symptomatic PHPT should be considered for parathyroidectomy, and identifies parathyroidectomy as an effective treatment to improve end organ complications, such as abnormal bone mineral density, renal stone disease and renal functions, and potentially cardiovascular outcomes and neurocognitive functions (Silverberg 2014). This consensus also recommends parathyroidectomy for the following groups with PHPT.

- People who develop the disease before the age of 50 years.

- Serum calcium levels greater than $1 \mathrm{mg} / \mathrm{dL}(0.25 \mathrm{mmol} / \mathrm{L})$ of the upper limit of normal laboratory reference range.

- Bone mineral density T-score of -2.5 or more at lumbar spine, femoral neck, total hip or distal $1 / 3^{\text {rd }}$ of radius for postmenopausal women or males greater than 50 years.

- Low trauma fragility fracture.

- A glomerular filtration rate of less than $60 \mathrm{~mL} / \mathrm{min}$, renal stones, nephrocalcinosis or high risk for renal stone formation in those with urine calcium of more than $400 \mathrm{mg} /$ day.

Parathyroidectomy is also associated with improvements in functional capacity and health-related quality of life even in asymptomatic individuals with PHPT (Ambrogini 2007; Morris 2010). Surgeons employ different techniques for parathyroidectomy depending on the type of involvement of the parathyroid glands. All individuals with the diagnosis of PHPT should be evaluated by parathyroid imaging studies in the attempt to localise the disease pathology in one or more of the parathyroid glands before considering surgical intervention. This approach enables the surgeons to decide the optimal surgical approach for parathyroidectomy. Localisation studies to identify the diseased parathyroid gland(s) include ultrasonography, nuclear medicine-based parathyroid scintigraphy, and multiphase or four-dimensional computed tomography (Kuzminski 2018). Minimally invasive parathyroidectomy is the ideal treatment option for patients with a well localised disease identified by preoperative parathyroid imaging (Khan 2017). Open surgery by parathyroid exploration becomes necessary among patients with non-localisation of adenoma(s) in parathyroid imaging. Although dependent on the surgical expertise, both focused, image-guided, minimally invasive parathyroidectomy and bilateral neck exploration can achieve high cure rates in people with PHPT (Wilhelm 2016).

\section{Minimally invasive parathyroidectomy}

Common approaches for minimally invasive parathyroidectomy involve open focal mini-incision (70\% of cases), video-assisted approach $(-20 \%$ of cases) or total endoscopic approach $(-10 \%$ of cases), depending on clinical situation and the surgeons' ability to perform the technique (Brunaud 2016; Taieb 2013). Gagner 1996 first described total endoscopic parathyroidectomy with subsequent refinements of the technique to the current day surgical practice. Video-assisted parathyroidectomy, first described in 1997 (Miccoli 1997), is also performed partially with the help of an endoscope but does not use gas to view the surgical field as in the other techniques. An extra-cervical endoscopic approach that avoids a neck scar, through the chest wall, breast, oral cavity, retro-auricular region and axilla, is also occasionally used for minimally invasive parathyroidectomy. Robotic parathyroidectomy that places a computer interface between patient and surgeon to optimise the feasibility and quality of surgery is a new approach in minimally invasive parathyroidectomy (Garas 2015). Intraoperative PTH monitoring helps the surgeons to verify successful surgery during minimally invasive parathyroidectomy. Intraoperative PTH monitoring involves measurement of $\mathrm{PTH}$, generally through peripheral veins, pre-incision, pre-gland ligation, and at 5, 10 and 20 minutes postgland ligation during the minimally invasive parathyroidectomy. A reduction in PTH level of more than $50 \%$ from the highest baseline value at 10 minutes postgland ligation is generally considered as evidence for adequate gland excision (Barczynski 2009). Intraoperative PTH monitoring has been recently reported to help prediction of successful parathyroidectomy with very high precision, and also to identify multiglandular disease and parathyroid cancer (Dobrinja 2017).

\section{Bilateral neck exploration for parathyroidectomy}

Until the late 1970s bilateral neck exploration surgical technique was the standard approach for parathyroidectomy in people with PHPT, which was successful in about $97 \%$ of cases and with reported complication rates of $1 \%$ to $2 \%$ (Brunaud 2016). The surgery involves exploration of all the four parathyroid glands to locate the abnormal gland (the adenoma) usually under general anaesthesia with a large neck incision. Bilateral neck exploration is now utilised only when preoperative localisation of the adenoma is 
not possible, in case of discordant localisation studies in parathyroid imaging, familial or genetic disorders of PHPT, and concomitant thyroid disease. The procedure may also be performed by a minimal incision or video-assisted endoscopic approach (Alesina 2013; Kreidieh 2013).

\section{Adverse effects of the intervention}

In general, parathyroidectomy is associated with low rates of surgical and postoperative complications. A recent systematic review of 82 observational studies and sic randomised controlled trials (RCTs) at moderate risk of bias concluded that minimally invasive parathyroidectomy and bilateral neck exploration are both effective techniques for parathyroidectomy in the treatment of PHPT, with the safety profile of minimally invasive parathyroidectomy being slightly superior to bilateral neck exploration (Singh 2016a). This publication also revealed a statistically significant increased risk of hypocalcaemia in the bilateral neck exploration group compared with the minimally invasive parathyroidectomy group ( $14 \%$ vs $2.3 \%, \mathrm{P}<0.001)$. Statistically significant lower risk of laryngeal nerve injury was also evident with minimally invasive parathyroidectomy compared to bilateral neck exploration $(0.3 \%$ vs $0.9 \%)$. However, the risk of infection $(0.5 \%$ vs $0.5 \%)$ and premature mortality $(0.1 \%$ vs $0.5 \%)$ were comparable in both groups. Another important complication of parathyroidectomy is hematoma formation that may present with neck pain, respiratory distress, dysphagia and increased drainage from the surgical wound (Kreidieh 2013). Open drainage of the hematoma may be necessary in some people. Hungry bone syndrome associated with severe postoperative hypocalcaemia requiring intense medical management can complicate some cases of severe PHPT after parathyroidectomy (Witteveen 2013). Although postoperative hypoparathyroidism with symptomatic hypocalcaemia is usually transient, some cases may warrant long-term follow-up and medical management (Fyrsten 2016).

Persistent PHPT with development of postoperative hypercalcaemia within six months of parathyroidectomy occurs in up to $5 \%$ of individuals because of incomplete removal of abnormal parathyroid tissue (Silverberg 2014). Recurrent disease, development of new onset PHPT, can also occur in up to $8 \%$ of individuals with sporadic parathyroid adenomas after 3 to 11 years. The abnormal glands are often located in eutopic positions (rather than an ectopic gland) that should be identified by localisation studies before reoperations (Khan 2017; Silverberg 2014). The reoperation is best performed as minimally invasive parathyroidectomy if the tumour is localised or as unilateral exploration on the other side that has not been explored previously. Reported success rate of reoperation exceeds $90 \%$ especially when preoperative localisation of the abnormal parathyroid tissue is possible and when parathyroidectomy is coupled with intraoperative PTH assay. However, postoperative complications including hypoparathyroidism are higher in people undergoing reoperation (Khan 2017; Silverberg 2014).

\section{How the intervention might work}

As PHPT is a consequence of excess production of PTH from one or more of the parathyroid glands, complete surgical removal of the abnormal parathyroid tissue is expected to cure the disease. Biochemical evidence of normalisation of raised PTH level with resolution of hypercalcaemia in people with PHPT after parathyroidectomy usually indicate cure of the disease. In individuals with normocalcaemic PHPT, normalisation of elevated PTH levels usually indicate cure. Regardless of the surgical technique used, high cure rates were observed in patients who underwent successful parathyroidectomy (Khan 2017; Kreidieh 2013; Silverberg 2014; Singh 2016a). Although there is a small risk of recurrence of the disease even in sporadic cases of PHPT, the health benefits of successful parathyroidectomy observed during postoperative followup period form clear rationale for recommending this treatment option for appropriately selected patients.

\section{Why it is important to do this review}

The prevalence of PHPT steadily increases with age, and older individuals tend to develop much more complications related to the disease compared to younger individuals (Oltmann 2014). Because of the availability of better healthcare, the proportion of elderly individuals has increased markedly in the global population in recent years. The cumulative risk of adverse hormonal effects from elevated PTH levels on target organs increase with the duration of PHPT, and older age-groups are more vulnerable to the end-organ complications related to the disease, such as osteoporosis, kidney disease, cognitive dysfunction and cardiovascular morbidity (Bilezikian 2017). Increased healthcare burden and the related economic loss are the consequences of such complications. Parathyroidectomy has been shown to improve bone-related health outcomes in PHPT in multiple observational studies and RCTs (Hansen 2012; Khosla 1999; Koumakis 2013; Lundstam 2015; Nilsson 2017; Vestergaard 2000). Other health benefits, such as prevention of deterioration of renal functions (Tassone 2015), improvement of cardiovascular morbidity (Best 2017; McMahon 2015), and better health-related quality of life (Aberg 2015), have been demonstrated following parathyroidectomy in patients with PHPT.

Although there are a few recent systematic reviews on this topic (Cheng 2015; McMahon 2015; Singh 2016a; Singh 2016b), and two previous Cochrane protocols suggest moderate health benefits of parathyroidectomy in people with PHPT in terms of improvements in bone mineral density, cognitive functions and healthrelated quality of life (Ramakant 2017; Smith 2017), there are still uncertainties on the overall health benefits of parathyroidectomy, the appropriate patient categories that benefit most from surgery, the severity of hyperparathyroidism for which parathyroidectomy should definitely be the choice, and the economic impact of surgery over medical therapy. Therefore, there is a need 
to compile the latest RCT evidence to examine the benefits of parathyroidectomy as a treatment option for the management of PHPT.

\section{O B JECT IVES}

To assess the effects of parathyroidectomy for adults with PHPT. We will also compare the effects of different types of parathyroidectomy on people with PHPT.

\section{METHODS}

\section{Criteria for considering studies for this review}

\section{Types of studies}

We will include RCTs.

\section{Types of participants}

We will limit participants of interest to adults (aged 18 years or more) with PHPT who underwent parathyroidectomy for the first time.

\section{Diagnostic criteria for PHPT}

We will define PHPT (Bilezikian 2017), by one or more of the following.

- Elevated or high normal PTH levels with elevated serum calcium levels above the normal laboratory reference values.

- Elevated serum PTH with normal calcium levels and exclusion of secondary causes for elevation of PTH (mainly decreased calcium intake, vitamin $\mathrm{D}$ deficiency, renal insufficiency and hypercalciuria of renal origin).

- Exclusion of secondary causes for hyperparathyroidism such as chronic renal insufficiency and vitamin D deficiency.

\section{Types of interventions}

We plan to investigate the following comparisons of intervention versus control/comparator.

\section{Intervention}

(a) Minimally invasive parathyroidectomy (focal mini-incision, video-assisted approach, totally-endoscopic or robotic approach). (b) Parathyroidectomy by bilateral surgical neck exploration.

\section{Comparator}

Participants compared with (a) or (b) managed by:

- biochemical, clinical and imaging follow-up without surgical interventions for any reason, such as personal preference, decision by treating physicians or contraindications for surgery from other serious medical conditions.

- pharmacotherapy such as bisphosphonates and calcium lowering drugs (calcimimetics) without parathyroidectomy.

We will investigate different parathyroidectomy surgical techniques with each other. Concomitant interventions must be the same in both the intervention and comparator groups to establish fair comparisons. If a trial includes multiple arms, we will include any trial arm that meets the inclusion criteria.

\section{Minimum duration of follow-up}

Minimal duration of follow-up will be six months after the surgical procedure. We will define any follow-up period going beyond the original time frame for the primary outcome measure as specified in the power calculation of the trial's protocol as an extended follow-up period (also called open-label extension study) (Buch 2011; Megan 2012).

\section{Summary of specific exclusion criteria}

We will exclude trials of the following category of participants.

- Participants with secondary hyperparathyroidism

- Participants with tertiary hyperparathyroidism

- Participants with different types of hyperparathyroidism with no separate reporting of results by type

- Duration of follow-up less than six months

- Trials in participants younger than 18 years

\section{Types of outcome measures}

We will not exclude a trial if it fails to report one or several of our primary or secondary outcome measures. If a trial does not report any of these primary or secondary outcomes, we will not include the trial but will provide some basic information in an additional table.

We will investigate the following outcomes using the methods and time points specified below.

\section{Primary outcomes}

- Cure of PHPT

- Morbidity related to PHPT

- Adverse events of surgery 
Secondary outcomes

- All-cause mortality

- Health-related quality of life

- Hospitalisation for correction of hypercalcaemia or acute renal impairment

- Socioeconomic effects

\section{Method of outcome measurement}

- Cure of PHPT: defined by improvement of hypercalcaemia and elevated PTH levels to the normal laboratory reference values.

- Morbidity related to PHPT such as improvement or lack of progression of osteoporosis osteopenia after parathyroidectomy, lack of progression of kidney dysfunction or renal stone disease, improvement or lack of progression of cognitive function, improvement or lack of worsening of cardiovascular disease.

- Adverse events of surgery: such as postoperative bleeding, infections, hypocalcaemia and speech problems.

- All-cause mortality: defined as death from any cause.

- Health-related quality of life: evaluated by a validated instrument such as medical outcomes study Short Form Survey 36 (SF-36);

- Hospitalisation for correction of severe hypercalcaemia (calcium level more than $3.0 \mathrm{mmol} / \mathrm{L}$ ) or acute renal impairment (requiring intravenous hydration or dialysis).

- Socioeconomic effects: such as direct costs defined as admission or readmission rates, average length of stay, visits to general practitioner, emergency hospital visits, medication consumption, indirect costs defined as resources lost due to illness by the participant or their family member.

\section{Timing of outcome measurement}

- Adverse events, all-cause mortality: measured at any time after participants were randomised to intervention or comparator groups.

- Health-related quality of life, socioeconomic effects: up to six months will be defined as short-term and longer than six months will be defined as long term.

- Cure of PHPT: measured by normal calcium and PTH levels at six months or later after participants were randomised to intervention or comparator groups.

- Morbidity related to PHPT: measured at six months or later after participants were randomised to intervention or comparator groups.

- Hospitalisation with hypercalcaemia or renal impairment: measured within six months after participants were randomised to intervention or comparator groups.

\section{Search methods for identification of studies}

\section{Electronic searches}

We will search the following sources from the inception of each database to the specified date and will place no restrictions on the language of publication.

- Cochrane Central Register of Controlled Trials

(CENTRAL) via the Cochrane Register of Studies Online (CRSO).

- MEDLINE Ovid (Epub Ahead of Print, In-Process \& Other Non-Indexed Citations, Ovid MEDLINE Daily and Ovid MEDLINE; from 1946 onwards).

- LILACS (Latin American and Caribbean Health Science Information database).

- ClinicalTrials.gov (www.clinicaltrials.gov).

- World Health Organization International Clinical Trials Registry Platform (WHO ICTRP) (www.who.int/trialsearch/).

We will not include Embase in our search, as RCTs indexed in Embase are now prospectively added to CENTRAL via a highly sensitive screening process (CENTRAL 2018).

We will continuously apply a MEDLINE (via Ovid SP) email alert service established by the Cochrane Metabolic and Endocrine Disorders (CMED) Group to identify newly published trials using the same search strategy as described for MEDLINE (Appendix 1). After we submit the review draft for editorial approval, the CMED Group will perform a complete search update on all databases available at the editorial office and will send the results to the review authors. Should we identify new trials for inclusion, we will evaluate these and incorporate the findings into our review (Beller 2013).

\section{Searching other resources}

We will try to identify other potentially eligible trials or ancillary publications by searching the reference lists of included trials, systematic reviews, meta-analyses and health technology assessment reports. In addition we will contact authors of included trials to identify any additional information on the retrieved trials and establish whether we may have missed further trials.

We will not use abstracts or conference proceedings for data extraction unless full data are available from trial authors because this information source does not fulfil the CONSORT requirements which consist of "an evidence-based, minimum set of recommendations for reporting randomised trials" (CONSORT 2016; Scherer 2007). We will present information on abstracts or conference proceedings in the 'Characteristics of studies awaiting classification' table.

\section{Data collection and analysis}




\section{Selection of studies}

After removal of duplicates, two review authors (JMP and AKV) will independently screen the abstract and title of every record retrieved by the searches. We will obtain the full-text of all potentially eligible relevant records, and will screen them for eligibility. We will resolve any disagreements through consensus or by recourse to a third review author (RS). If we cannot resolve a disagreement, we will categorise the trial as a 'study awaiting classification' and will contact the trial authors for clarification. We will present an adapted PRISMA flow diagram to show the process of trial selection (Liberati 2009). We will list all articles excluded after full-text assessment in a 'Characteristics of excluded studies' table and will provide the reasons for exclusion.

\section{Data extraction and management}

Two review authors (JMP and RS) will independently extract the data from trials that fulfil our inclusion criteria to identify key participant and intervention characteristics. We will describe interventions by use of the 'template for intervention description and replication' (TIDieR) checklist (Hoffmann 2014; Hoffmann 2017).

We will report data on efficacy outcomes and adverse events using standardised data extraction sheets from the CMED Group. We will resolve any disagreements by discussion or, if required, we will consult a third review author (AKV).

We will provide information about potentially relevant ongoing trials, including the trial identifiers, in the table 'Characteristics of ongoing trials' and in a joint appendix 'Matrix of trial endpoint (publications and trial documents)'. We will try to find the protocol for each included trial and we will report primary, secondary and other outcomes in comparison with data in publications in a joint appendix.

We will email all authors of included trials to enquire whether they would be willing to answer questions regarding their trials. We will present the results of this survey in an appendix. We will thereafter seek relevant missing information on the trial from the primary trial author(s), if required.

\section{Dealing with duplicate and companion publications}

In the event of duplicate publications, companion documents or multiple reports of a primary trial, we will maximise the information yield by collating all available data, and we will use the most complete data set aggregated across all known publications. We will list duplicate publications, companion documents, multiple reports of a primary trial, and trial documents of included trials (such as trial registry information) as secondary references under the study ID of the included trial. Furthermore, we will also list duplicate publications, companion documents, multiple reports of a trial, and trial documents of excluded trials (such as trial registry information) as secondary references under the study ID of the excluded trial.

\section{Data from clinical trials registers}

If data from included trials are available as study results in clinical trials registers, such as ClinicalTrials.gov or similar sources, we will make full use of this information and will extract the data. If there is also a full publication of the trial, we will collate and critically appraise all available data. If an included trial is marked as a completed study in a clinical trial register but no additional information (study results, publication or both) is available, we will add this trial to the 'Characteristics of studies awaiting classification' table.

\section{Assessment of risk of bias in included studies}

Two review authors (JMP and IML) will independently assess the risk of bias of each included trial. We will resolve any disagreements by consensus or by consulting a third review author (AKV). In the case of disagreement, we will consult the rest of the review author team and we will make a judgement based on consensus. If adequate information is unavailable from the publications, trial protocols or other sources, we will contact the trial authors for more detail to request missing data on 'Risk of bias' items.

We will use the Cochrane 'Risk of bias' assessment tool (Higgins 2017), assigning assessments of low, high or unclear risk of bias (for details see Appendix 2; Appendix 3). We will evaluate individual bias items as described in the Cochrane Handbook for Systematic Reviews of Interventions according to the criteria and associated categorisations contained therein (Higgins 2017).

\section{Summary assessment of risk of bias}

We will present a 'Risk of bias' graph and a 'Risk of bias' summary figure.

We will distinguish between self-reported, investigator-assessed and adjudicated outcome measures.

We will consider the following self-reported outcomes.

- Health-related quality of life

- Adverse events of surgery

We will consider the following outcomes to be investigator-assessed.

- Cure of PHPT

- Morbidity related to PHPT

- All-cause mortality

- Adverse events of surgery

- Hospitalisation with hypercalcaemia or renal impairment

- Socioeconomic effects

\section{Risk of bias for a trial across outcomes}

Some risk of bias domains, such as selection bias (sequence generation and allocation sequence concealment), affect the risk of bias across all outcome measures in a trial. In the case of high risk of 
selection bias, we will mark all endpoints investigated in the associated trial as being at high risk. Otherwise, we will not perform a summary assessment of the risk of bias across all outcomes for a trial.

\section{Risk of bias for an outcome within a trial and across domains}

We will assess the risk of bias for an outcome measure by including all entries relevant to that outcome (i.e. both trial-level entries and outcome-specific entries). We consider low risk of bias to denote a low risk of bias for all key domains, unclear risk to denote an unclear risk of bias for one or more key domains and high risk to denote a high risk of bias for one or more key domains.

\section{Risk of bias for an outcome across trials and across domains}

These are the main summary assessments that we will incorporate into our judgments about the certainty of evidence in the 'Summary of findings' tables. We will define outcomes as at low risk of bias when most information comes from trials at low risk of bias, unclear risk when most information comes from trials at low or unclear risk of bias, and high risk when a sufficient proportion of information comes from trials at high risk of bias.

\section{Measures of treatment effect}

When at least two included trials are available for a comparison and a given outcome, we will try to express dichotomous data as a risk ratio (RR) or odds ratio (OR) with $95 \%$ confidence intervals (CIs). For continuous outcomes measured on the same scale (e.g. weight loss in $\mathrm{kg}$ ), we will estimate the intervention effect using the mean difference (MD) with 95\% CIs. For continuous outcomes that measure the same underlying concept (e.g. health-related quality of life) but use different measurement scales, we will calculate the standardised mean difference (SMD). We will express time-toevent data as a hazard ratio (HR) with 95\% CIs.

\section{Unit of analysis issues}

We will take into account the level at which randomisation occurred, such as for cross-over trials, cluster-randomised trials and multiple observations for the same outcome. If more than one comparison from the same trial is eligible for inclusion in the same meta-analysis, we will either combine groups to create a single pair-wise comparison or appropriately reduce the sample size so that the same participants do not contribute data to the metaanalysis more than once (splitting the 'shared' group into two or more groups). While the latter approach offers some solution to adjusting the precision of the comparison, it does not account for correlation arising from the same set of participants being in multiple comparisons (Higgins 2011).
We will attempt to re-analyse cluster-RCTs that have not appropriately adjusted for potential clustering of participants within clusters in their analyses. The variance of the intervention effects will be inflated by a design effect. Calculation of a design effect involves estimation of an intracluster correlation coefficient (ICC). We will obtain estimates of ICCs by contacting trial authors or by imputing the ICC values by using either estimates from other included trials that report ICCs or external estimates from empirical research (e.g. Bell 2013). We plan to examine the impact of clustering using sensitivity analyses.

\section{Dealing with missing data}

If possible, we will obtain missing data from the authors of the included trials. We will carefully evaluate important numerical data such as screened, randomly assigned participants as well as intention-to-treat, and as-treated and per-protocol populations. We will investigate attrition rates (e.g. dropouts, losses to followup, withdrawals), and we will critically appraise issues concerning missing data and use of imputation methods (e.g. last observation carried forward).

In trials where the standard deviation (SD) of the outcome is not available at follow-up or we cannot recreate it, we will standardise by the mean of the pooled baseline SD from those trials that reported this information.

Where included trials do not report means and SDs for outcomes and we do not receive the necessary information from trial authors, we will impute these values by estimating the mean and variance from the median, range and the size of the sample (Hozo 2005). We will investigate the impact of imputation on meta-analyses by performing sensitivity analyses, and we will report for every outcome which trials had imputed SDs.

\section{Assessment of heterogeneity}

In the event of substantial clinical or methodological heterogeneity, we will not report trial results as the pooled effect estimate in a meta-analysis.

We will identify heterogeneity (inconsistency) by visually inspecting the forest plots and by using a standard $\mathrm{Chi}^{2}$ test with a significance level of $\alpha=0.1$ (Deeks 2017). In view of the low power of this test, we will also consider the $\mathrm{I}^{2}$ statistic, which quantifies inconsistency across trials to assess the impact of heterogeneity on the meta-analysis (Higgins 2002; Higgins 2003).

When we find heterogeneity, we will attempt to determine the possible reasons for it by examining individual trial and subgroup characteristics.

\section{Assessment of reporting biases}

If we include 10 or more trials that investigate a particular outcome, we will use funnel plots to assess small-trial effects. Several explanations may account for funnel plot asymmetry, including 
true heterogeneity of effect with respect to trial size, poor methodological design (and hence bias of small trials) and publication bias (Sterne 2017). Therefore we will interpret the results carefully (Sterne 2011).

\section{Data synthesis}

We plan to undertake (or display) a meta-analysis only if we judge participants, interventions, comparisons and outcomes to be sufficiently similar to ensure an answer that is clinically meaningful. Unless good evidence shows homogeneous effects across trials of different methodological quality, we will primarily summarise low risk of bias data using a random-effects model (Wood 2008). We will interpret random-effects meta-analyses with due consideration to the whole distribution of effects and present a prediction interval (Borenstein 2017a; Borenstein 2017b; Higgins 2009). A prediction interval needs at least four trials to be calculated and specifies a predicted range for the true treatment effect in an individual trial (Riley 2011). For rare events such as event rates below $1 \%$, we will use the Peto's odds ratio method provided that there is no substantial imbalance between intervention and comparator group sizes and intervention effects are not exceptionally large. In addition, we will perform statistical analyses according to the statistical guidelines presented in the Cochrane Handbook for Systematic Reviews of Interventions (Deeks 2017).

\section{Subgroup analysis and investigation of heterogeneity}

We expect the following characteristics to introduce clinical heterogeneity, and we plan to carry out the following subgroup analyses including investigation of interactions (Altman 2003).

- Different surgical techniques

- Severity of PHPT

- Difference in the demographics of trial population such as gender, age and ethnic differences

\section{Sensitivity analysis}

We plan to perform sensitivity analyses to explore the influence of the following factors (when applicable) on effect sizes by restricting analysis to the following.

- Published trials.

- Effect of risk of bias, as specified in the 'Assessment of risk of bias in included studies' section.

- Very long or large trials to establish the extent to which they dominate the results.

- Using the following filters: diagnostic criteria, imputation, language of publication, source of funding (industry versus other) or country.

We will also test the robustness of results by repeating the analyses using different measures of effect size (RR, OR, etc.) and different statistical models (fixed-effect and random-effects models).

\section{Certainty of the evidence}

We will present the overall certainty of evidence for each outcome specified below, according to the GRADE approach, which takes into account issues related not only to internal validity (risk of bias, inconsistency, imprecision, publication bias) but also to external validity, such as directness of results. Two review authors (JMP and IML) will independently assess the certainty of evidence for each outcome. We will resolve any differences in assessment by discussion or consulting a third review author (RS).

We will include an appendix entitled 'Checklist to aid consistency and reproducibility of GRADE assessments', to help with standardisation of the 'Summary of findings' tables (Meader 2014). Alternatively, we will use the GRADEpro Guideline Development Tool (GDT) software and will present evidence profile tables as an appendix (GRADEpro GDT 2015). We will present results for the outcomes as described in the 'Types of outcome measures' section. If meta-analysis is not possible, we will present the results in a narrative format in the 'Summary of findings' table. We will justify all decisions to downgrade the quality of trials using footnotes, and we will make comments to aid the reader's understanding of the Cochrane Review where necessary.

\section{'Summary of findings' table}

We will present a summary of the evidence in a 'Summary of findings' table. This will provide key information about the best estimate of the magnitude of the effect, in relative terms and as absolute differences, for each relevant comparison of alternative management strategies, numbers of participants and trials addressing each important outcome and a rating of overall confidence in effect estimates for each outcome. We will create the 'Summary of findings' table based on the methods described in the Cochrane Handbook for Systematic Reviews of Interventions (Schünemann 2017) using Review Manager 5 (RevMan 5) table editor (RevMan 2014). We will report the following outcomes, listed according to priority.

- Cure of PHPT

- Morbidity related to PHPT

- All-cause mortality

- Adverse events of surgery

- Health-related quality of life

- Hospitalisation with hypercalcaemia or renal impairment

- Socioeconomic effects.

\section{ACKNOWLEDGEMENTS}

We thank the CMED Information Specialist, Maria-Inti Metzendorf, for developing the search strategies. 


\section{REFERE N C E S}

\section{Additional references}

\section{Aberg 2015}

Aberg V, Norenstedt S, Zedenius J, Sääf M, Nordenström J, Pernow $Y$, et al. Health-related quality of life after successful surgery for primary hyperparathyroidism: no additive effect from vitamin $\mathrm{D}$ supplementation: results of a double-blind randomized study. European Journal of Endocrinology 2015; 172(2):181-7.

\section{Alesina 2013}

Alesina PF, Hinrichs J, Heuer M, Hofmeister S, Meier B, Walz MK. Feasibility of video-assisted bilateral neck exploration for patients with primary hyperparathyroidism and failed or discordant localization studies. Langenbeck's Archives of Surgery 2013;398(1):107-11.

Altman 2003

Altman DG, Bland JM. Interaction revisited: the difference between two estimates. BMJ 2003;326(7382):219. [PUBMED: 12543843]

Ambrogini 2007

Ambrogini E, Cetani F, Cianferotti L, Vignali E, Banti C, Viccica G, et al. Surgery or surveillance for mild asymptomatic primary hyperparathyroidism: a prospective, randomized clinical trial. Journal of Clinical Endocrinology and Metabolism 2007;92(8):3114-21.

Barczynski 2009

Barczynski M, Konturek A, Hubalewska-Dydejczyk A, Cichon S, Nowak W. Evaluation of Halle, Miami, Rome, and Vienna intraoperative iPTH assay criteria in guiding minimally invasive parathyroidectomy. Langenbeck's Archives of Surgery 2009;394(5):843-9.

Bell 2013

Bell ML, McKenzie JE. Designing psycho-oncology randomised trials and cluster randomised trials: variance components and intra-cluster correlation of commonly used psychosocial measures. Psychooncology 2013;22(8): 1738-47.

Beller 2013

Beller EM, Chen JK, Wang UL, Glasziou PP. Are systematic reviews up-to-date at the time of publication?. Systematic Reviews 2013;2:36. [2046-4053: (Electronic)]

Best 2017

Best CAE, Krishnan R, Malvankar-Mehta MS, MacNeil $\mathrm{SD}$. Echocardiogram changes following parathyroidectomy for primary hyperparathyroidism: a systematic review and meta-analysis. Medicine 2017;96(43):e7255.

Bilezikian 2017

Bilezikian JP, Bandeira L, Khan A, Cusano NE. Hyperparathyroidism. Lancet 2018;391(10116):168-78. [PUBMED: 28923463]

\section{Borenstein 2017a}

Borenstein M, Higgins JP, Hedges LV, Rothstein HR Basics of meta-analysis: $\mathrm{I}^{2}$ is not an absolute measure of heterogeneity. Research Synthesis Methods 2017;8(1):5-18.
Borenstein 2017b

Borenstein M. Prediction intervals. www.metaanalysis.com/prediction (accessed 3 July 2017).

\section{Boutron 2014}

Boutron I, Altman DG, Hopewell S, Vera-Badillo F, Tannock I, Ravaud P. Impact of spin in the abstracts of articles reporting results of randomized controlled trials in the field of cancer: the SPIIN randomized controlled trial. Journal of Clinical Oncology 2014;32(36):4120-6.

\section{Brunaud 2016}

Brunaud L, Li Z, Van Den Heede K, Cuny T, Van Slycke $S$. Endoscopic and robotic parathyroidectomy in patients with primary hyperparathyroidism. Gland Surgery 2016;5 (3):352-60

\section{Buch 2011}

Buch MH, Aletaha D, Emery P, Smolen JS. Reporting of long-term extension studies: lack of consistency calls for consensus. Annals of the Rheumatic Diseases 2011;70(6): 886-90.

\section{CENTRAL 2018}

CENTRAL creation details. www.cochranelibrary.com/ help/central-creation-details.html (accessed 15 March 2018).

\section{Cheng 2015}

Cheng SP, Lee JJ, Liu TP, Yang PS, Liu SC, Hsu YC, et al. Quality of life after surgery or surveillance for asymptomatic primary hyperparathyroidism: a metaanalysis of randomized controlled trials. Medicine 2015;94 (23): 931.

\section{Cipriani 2015}

Cipriani C, Biamonte F, Costa AG, Zhang C, Biondi P, Diacinti $\mathrm{D}$, et al. Prevalence of kidney stones and vertebral fractures in primary hyperparathyroidism using imaging technology. Journal of Clinical Endocrinology and Metabolism 2015;100(4):1309-15.

\section{CONSORT 2016}

The CONSORT statement. www.consort-statement.org (accessed 19 May 2016).

\section{Corbett 2014}

Corbett MS, Higgins JP, Woolacott NF. Assessing baseline imbalance in randomised trials: implications for the Cochrane risk of bias tool. Research Synthesis Methods 2014; 5(1):79-85.

\section{Deeks 2017}

Deeks JJ, Higgins JPT, Altman DG (editors) on behalf of the Cochrane Statistical Methods Group. Chapter 9: Analysing data and undertaking meta-analyses. In: Higgins JPT, Churchill R, Chandler J, Cumpston MS (editors), Cochrane Handbook for Systematic Reviews of Interventions Version 5.2.0 (updated June 2017), Cochrane, 2017. Available from training.cochrane.org/handbook. 


\section{Dobrinja 2017}

Dobrinja C, Santandrea G, Giacca M, Stenner E, Ruscio M, de Manzini N. Effectiveness of intraoperative parathyroid monitoring (ioPTH) in predicting a multiglandular or malignant parathyroid disease. International Journal of Surgery (London, England) 2017;41(Suppl 1):S26-33.

\section{Fyrsten 2016}

Fyrsten E, Norlén O, Hessman O, Stålberg P, Hellman P. Long-term surveillance of treated hyperparathyroidism for multiple endocrine neoplasia type 1: recurrence or hypoparathyroidism?. World Journal of Surgery 2016;40(3): 615-21.

Gagner 1996

Gagner M. Endoscopic subtotal parathyroidectomy in patients with primary hyperparathyroidism. British Journal of Surgery 1996;83(6):875.

\section{Garas 2015}

Garas G, Holsinger FC, Grant DG, Athanasiou T, Arora A, Tolley N. Is robotic parathyroidectomy a feasible and safe alternative to targeted open parathyroidectomy for the treatment of primary hyperparathyroidism?. International Journal of Surgery (London, England) 2015;15:55-60.

GRADEpro GDT 2015 [Computer program] GRADE Working Group, McMaster University. GRADEpro GDT. Version accessed 19 April 2018. Hamilton (ON): GRADE Working Group, McMaster University, 2015.

\section{Hansen 2012}

Hansen S, Hauge EM, Rasmussen L, Jensen JE, Brixen K. Parathyroidectomy improves bone geometry and microarchitecture in female patients with primary hyperparathyroidism: a one-year prospective controlled study using high-resolution peripheral quantitative computed tomography. Journal of Bone and Mineral Research 2012;27(5):1150-8.

\section{Higgins 2002}

Higgins JPT, Thompson SG. Quantifying heterogeneity in a meta-analysis. Statistics in Medicine 2002;21(11):1539-58.

\section{Higgins 2003}

Higgins JPT, Thompson SG, Deeks JJ, Altman DG. Measuring inconsistency in meta-analysis. BMJ 2003;327 (7414):557-60.

\section{Higgins 2009}

Higgins JPT, Thompson SG, Spiegelhalter DJ. A reevaluation of random-effects meta-analysis. Journal of the Royal Statistical Society: Series A (Statistics in Society) 2009; 172(1):137-59.

\section{Higgins 2011}

Higgins JPT, Deeks JJ, Altman DG. Chapter 16: Special topics in statistics. In: Higgins JPT, Green S, editor (s). Cochrane Handbook for Systematic Reviews of Interventions Version 5.1.0 (updated March 2011). The Cochrane Collaboration, 2011. Available from handbook.cochrane.org.

\section{Higgins 2017}

Higgins JPT, Altman DG, Sterne JAC (editors). Chapter 8: Assessing risk of bias in included studies. In: Higgins JPT, Churchill R, Chandler J, Cumpston MS (editors), Cochrane Handbook for Systematic Reviews of Interventions Version 5.2.0 (updated June 2017), Cochrane, 2017. Available from training.cochrane.org/handbook.

\section{Hoffmann 2014}

Hoffmann TC, Glasziou PP, Boutron I, Milne R, Perera R, Moher D, et al. Better reporting of interventions: template for intervention description and replication (TIDieR) checklist and guide. BMJ 2014;348:g1687.

\section{Hoffmann 2017}

Hoffmann TC, Oxman AD, Ioannidis JP, Moher D, Lasserson TJ, Tovey DI, et al. Enhancing the usability of systematic reviews by improving the consideration and description of interventions. BMJ 2017;358:;2998.

\section{Hozo 2005}

Hozo SP, Djulbegovic B, Hozo I. Estimating the mean and variance from the median, range, and the size of a sample. BMC Medical Research Methodology 2005;5:13. DOI: 10.1186/1471-2288-5-13

\section{Hróbjartsson 2013}

Hróbjartsson A, Thomsen AS, Emanuelsson F, Tendal B, Hilden J, Boutron I, et al. Observer bias in randomized clinical trials with measurement scale outcomes: a systematic review of trials with both blinded and nonblinded assessors. Canadian Medical Association Journal 2013;185 (4):E201-11.

Jones 2015 Jones CW, Keil LG, Holland WC, Caughey MC, PlattsMills TF. Comparison of registered and published outcomes in randomized controlled trials: a systematic review. $B M C$ Medicine 2015;13:282. DOI: 10.1186/s12916-015-0520-3

Khan 2017

Khan AA, Hanley DA, Rizzoli R, Bollerslev J, Young JE, Rejnmark L, et al. Primary hyperparathyroidism: review and recommendations on evaluation, diagnosis, and management. A Canadian and international consensus. Osteoporosis International 2017;28(1):1-19. [PUBMED: 27613721]

\section{Khosla 1999}

Khosla S, Melton LJ 3rd, Wermers RA, Crowson CS, O'Fallon W, Riggs B. Primary hyperparathyroidism and the risk of fracture: a population-based study. Journal of Bone and Mineral Research 1999;14(10):1700-7.

\section{Kirkham 2010}

Kirkham JJ, Dwan KM, Altman DG, Gamble C, Dodd $S$, Smyth R, et al. The impact of outcome reporting bias in randomised controlled trials on a cohort of systematic reviews. BMJ 2010;340:c365. DOI: 10.1136/bmj.c365

\section{Koumakis 2013}

Koumakis E, Souberbielle JC, Sarfati E, Meunier M, Maury E, Gallimard E, et al. Bone mineral density evolution after successful parathyroidectomy in patients 
with normocalcemic primary hyperparathyroidism. Journal of Clinical Endocrinology and Metabolism 2013;98(8): 3213-20.

\section{Kreidieh 2013}

Kreidieh OI, Ahmadieh H, Akl EA, El-Hajj FG. Minimally invasive parathyroidectomy guided by intraoperative parathyroid hormone monitoring (IOPTH) and preoperative imaging versus bilateral neck exploration for primary hyperparathyroidism in adults. Cochrane Database of Systematic Reviews 2013, Issue 10. DOI: 10.1002/ 14651858.CD010787

Kuzminski 2018

Kuzminski SJ, Sosa JA, Hoang JK. Update in parathyroid imaging. Magnetic Resonance Imaging Clinics of North America 2018;26(1):151-66.

\section{Liberati 2009}

Liberati A, Altman DG, Tetzlaff J, Mulrow C, Gøtzsche PC, Ioannidis JPA, et al. The PRISMA statement for reporting systematic and meta-analyses of studies that evaluate interventions: explanation and elaboration. PLOS Medicine 2009;6(7):e1000100. DOI: 10.1371/ journal.pmed. 1000100

\section{Lundstam 2015}

Lundstam K, Heck A, Mollerup C, Godang K, Baranowski $\mathrm{M}$, Pernow $\mathrm{Y}$, et al. Effects of parathyroidectomy versus observation on the development of vertebral fractures in mild primary hyperparathyroidism. Journal of Clinical Endocrinology and Metabolism 2015;100(4):1359-67.

\section{Mathieu 2009}

Mathieu S, Boutron I, Moher D, Altman DG, Ravaud P. Comparison of registered and published primary outcomes in randomized controlled trials. JAMA 2009;302:977-84.

\section{McMahon 2015}

McMahon DJ, Carrelli A, Palmeri N, Zhang C, DiTullio M, Silverberg SJ, et al. Effect of parathyroidectomy upon left ventricular mass in primary hyperparathyroidism: a metaanalysis. Journal of Clinical Endocrinology and Metabolism 2015;100(12):4399-407.

\section{Meader 2014}

Meader N, King K, Llewellyn A, Norman G, Brown J, Rodgers $\mathrm{M}$, et al. A checklist designed to aid consistency and reproducibility of GRADE assessments: development and pilot validation. Systematic Reviews 2014;3:82.

\section{Megan 2012}

Megan B, Pickering RM, Weatherall M. Design, objectives, execution and reporting of published open-label extension studies. Journal of Evaluation in Clinical Practice 2012;18 (2):209-15.

\section{Miccoli 1997}

Miccoli P, Pinchera A, Cecchini G, Conte M, Bendinelli C, Vignali E, et al. Minimally invasive, video-assisted parathyroid surgery for primary hyperparathyroidism. Journal of Endocrinological Investigation 1997;20(7):429-30.

\section{Morris 2010}

Morris GS, Grubbs EG, Hearon CM, Gantela S, Lee JE, Evans DB, et al. Parathyroidectomy improves functional capacity in "asymptomatic" older patients with primary hyperparathyroidism: a randomized control trial. Annals of Surgery 2010;251(5):832-7. [PUBMED: 20395857]

Nilsson 2017

Nilsson IL, Norenstedt S, Zedenius J, Pernow Y, Bränström R. Primary hyperparathyroidism, hypercalciuria, and bone recovery after parathyroidectomy. Surgery 2017;162(2): 429-36.

Ning 2009

Ning L, Sippel R, Schaefer S, Chen H. What is the clinical significance of an elevated parathyroid hormone level after curative surgery for primary hyperparathyroidism?. Annals of Surgery 2009;249(3):469-72.

\section{Oltmann 2014}

Oltmann SC, Rajaei MH, Sippel RS, Chen H, Schneider DF. Primary hyperparathyroidism across the ages: presentation and outcomes. Journal of Surgical Research 2014;190(1):185-90.

Pepe 2017

Pepe J, Cipriani C, Sonato C, Raimo O, Biamonte F, Minisola S. Cardiovascular manifestations of primary hyperparathyroidism: a narrative review. European Journal of Endocrinology 2017;177(6):R297-308.

\section{Ramakant 2017}

Ramakant P, Paul MJ, Paul TV, Rao SD, Abraham DT, Uttley L, et al. Surgery versus surveillance for asymptomatic (mild) primary hyperparathyroidism in adults 50 years or older. Cochrane Database of Systematic Reviews 2017, Issue 7. DOI: $10.1002 / 14651858 . C D 010093 . p u b 2$

\section{RevMan 2014 [Computer program]}

Nordic Cochrane Centre, The Cochrane Collaboration. Review Manager 5 (RevMan 5). Version 5.3. Copenhagen: Nordic Cochrane Centre, The Cochrane Collaboration, 2014.

\section{Riley 2011}

Riley RD, Higgins JP, Deeks JJ. Interpretation of random effects meta-analyses. BMJ 2011;342:d549.

\section{Scherer 2007}

Scherer RW, Langenberg P, von Elm E. Full publication of results initially presented in abstracts. Cochrane Database of Systematic Reviews 2007, Issue 2. DOI: 10.1002/ 14651858.MR000005.pub3

\section{Schünemann 2017}

Schünemann HJ, Oxman AD, Higgins JPT, Vist GE, Glasziou P, Akl E, et al. on behalf of the Cochrane GRADEing Methods Group and the Cochrane Statistical Methods Group. Chapter 11: Completing 'Summary of findings' tables and grading the confidence in or quality of the evidence. In: Higgins JPT, Churchill R, Chandler J, Cumpston MS (editors), Cochrane Handbook for Systematic Reviews of Interventions Version 5.2.0 
(updated June 2017). Cochrane, 2017. Available from training.cochrane.org/handbook.

\section{Silverberg 2014}

Silverberg SJ, Clarke BL, Peacock M, Bandeira F, Boutroy $S$, Cusano NE, et al. Current issues in the presentation of asymptomatic primary hyperparathyroidism: proceedings of the fourth international workshop. Journal of Clinical Endocrinology and Metabolism 2014;99(10):3580-94.

\section{Singh 2016a}

Singh Ospina N, Maraka S, Rodriguez-Gutierrez R, Espinosa de Ycaza AE, Jasim S, Gionfriddo M, et al. Comparative efficacy of parathyroidectomy and active surveillance in patients with mild primary hyperparathyroidism: a systematic review and metaanalysis. Osteoporosis International 2016;27(12):3395-407.

\section{Singh 2016b}

Singh Ospina NM, Rodriguez-Gutierrez R, Maraka S, Espinosa de Ycaza AE, Jasim S, Castaneda-Guarderas A, et al. Outcomes of parathyroidectomy in patients with primary hyperparathyroidism: a systematic review and meta-analysis. World Journal of Surgery 2016;40(10): 2359-77.

\section{Smith 2017}

Smith ME, Pfleiderer AG, Shamil E. Open minimally invasive versus video-assisted minimally invasive parathyroidectomy for primary hyperparathyroidism. Cochrane Database of Systematic Reviews 2017, Issue 1. DOI: $10.1002 / 14651858 . C D 012512$

\section{Sterne 2011}

Sterne JA, Sutton AJ, Ioannidis JP, Terrin N, Jones DR, Lau $\mathrm{J}$, et al. Recommendations for examining and interpreting funnel plot asymmetry in meta-analyses of randomised controlled trials. BMJ 2011;343:d4002.

\section{Sterne 2017}

Sterne JAC, Egger M, Moher D, Boutron I (editors). Chapter 10: Addressing reporting biases. In: Higgins JPTG, Churchill R, Chandler J, Cumpston MS (editors), Cochrane Handbook for Systematic Reviews of Interventions Version 5.2.0 (updated June 2017), Cochrane, 2017. Available from training.cochrane.org/handbook.

\section{Taieb 2013}

Taieb A, Seman M, Menegaux F, Tresallet C. Surgical technique parathyroidectomy through a minimally invasive gland-centred localized approach for primary hyperparathyroidism. Journal of Visceral Surgery 2013;150 (6):403-6.

\section{Tassone 2015}

Tassone F, Guarnieri A, Castellano E, Baffoni C, Attanasio R, Borretta G. Parathyroidectomy halts the deterioration of renal function in primary hyperparathyroidism. Journal of Clinical Endocrinology and Metabolism 2015;100(8): 3069-73.

\section{Trombetti 2016}

Trombetti A, Christ ER, Henzen C, Gold G, Brändle M, Herrmann FR, et al. Clinical presentation and management of patients with primary hyperparathyroidism of the Swiss primary hyperparathyroidism cohort: a focus on neuro-behavioral and cognitive symptoms. Journal of Endocrinological Investigation 2016;39(5):567-76.

\section{Verdelli 2017}

Verdelli C, Corbetta S. Mechanisms in endocrinology: kidney involvement in patients with primary hyperparathyroidism: an update on clinical and molecular aspects. European Journal of Endocrinology 2017;176(1): R39-52.

\section{Vestergaard 2000}

Vestergaard P, Mollerup CL, Frokjaer VG, Christiansen P, Blichert-Toft M, Mosekilde L. Cohort study of risk of fracture before and after surgery for primary hyperparathyroidism. BMJ (Clinical research ed) 2000;321 (7261):598-602.

\section{Wilhelm 2016}

Wilhelm SM, Wang TS, Ruan DT, Lee JA, Asa SL, Duh QY, et al. The American Association of Endocrine Surgeons guidelines for definitive management of primary hyperparathyroidism. JAMA Surgery 2016;151(10): 959-68.

\section{Witteveen 2013}

Witteveen JE, van Thiel S, Romijn JA, Hamdy NA. Hungry bone syndrome: still a challenge in the post-operative management of primary hyperparathyroidism: a systematic review of the literature. European Journal of Endocrinology 2013;168(3):R45-53.

\section{Wood 2008}

Wood L, Egger M, Gluud LL, Schulz KF, Jüni P, Altman DG, et al. Empirical evidence of bias in treatment effect estimates in controlled trials with different interventions and outcomes: meta-epidemiological study. BMJ 2008;336 (7644):601-5.

\section{Yener 2016}

Yener Ozturk F, Erol S, Canat MM, Karatas S, Kuzu I, Dogan Cakir $S$, et al. Patients with normocalcemic primary hyperparathyroidism may have similar metabolic profile as hypercalcemic patients. Endocrine Journal 2016;63(2): 111-8.

* Indicates the major publication for the study 


\section{A P P E N D I C ES}

\section{Appendix I. Search strategies}

\section{MEDLINE (Ovid SP)}

1. Hyperparathyroidism, Primary/

2. (primary adj3 hyperparathyroid*).tw.

3. Parathyroid Neoplasms/

4. (parathyroid adj3 (adenoma? or hyperplasia? or neoplasm? or tumo?r?)).tw

5. or/1-4

6. Parathyroidectomy/

7. parathyroidectom*.tw.

8. (parathyroid* adj6 (surger* or surgic* or resection or excision or exploration)).tw

9. ((endoscop* or video or camera or minimally invasive or small incision or open) adj6 (surger* or surgic* or resection or excision or exploration)).tw

10. or/6-9

11.5 and 10

[Cochrane Handbook 2008 RCT filter - sensitivity maximizing version]

12. randomized controlled trial.pt.

13. controlled clinical trial.pt.

14. randomi?ed.ab.

15. placebo.ab.

16. drug therapy.fs.

17. randomly.ab.

18. trial.ab.

19. groups.ab.

20. or/12-19

21. exp animals/ not humans/

22. 20 not 21

23. 11 and 22

\section{CENTRAL (Cochrane Library)}

1. [mh "Hyperparathyroidism, Primary"]

2. (primary near/4 hyperparathyroid*):ti,ab,kw

3. [mh "Parathyroid Neoplasms"]

4. (parathyroid near/4 (adenoma* or hyperplasia* or neoplasm* or tumor* or tumour*)):ti,ab,kw

5. $\{$ or \#1-\#4\}

6. [mh "Parathyroidectomy"]

7. parathyroidectom*:ti,ab,kw

8. (parathyroid* near/7 (surgic* or resection or excision or exploration)):ti,ab,kw

9. ((endoscop* ${ }^{*}$ or video or camera or minimally invasive or small incision or open) near/7 (surgic* or resection or excision or exploration)):ti,ab,kw

10. surger*:ti,ab,kw

11. $\{$ or \#6-\#10\}

12. \#5 and \#11

\section{LILACS (iAHx)}

Parathyroidectomy for adults with primary hyperparathyroidism (Protocol)

Copyright ( 2018 The Cochrane Collaboration. Published by John Wiley \& Sons, Ltd. 
(MH: "Hyperparathyroidism, Primary" OR hyperparathyroid\$ OR hiperparatireoid\$ OR MH:"Parathyroid Neoplasms" OR (parathyroid AND (adenoma or hyperplasia \$ or neoplasm\$ or tumor\$))) AND (MH:"Parathyroidectomy" OR parathyroidectom\$ OR paratiroidectom\$ OR cirur\$ OR surger\$ or surgic\$ OR resection OR excision OR exploration) + Filter "Controlled Clinical Trial"

\section{WHO ICTRP (Advanced search)}

hyperparathyroid* AND parathyroidectom* OR

hyperparathyroid* AND surg* OR

parathyroid AND parathyroidectom* OR

parathyroid AND surg*

ClinicalTrials.gov (Advanced search)

Condition or disease: hyperparathyroidism OR parathyroid

Intervention/treatment: parathyroidectomy OR surgery OR surgical OR resection OR exploration

\section{Appendix 2. 'Risk of bias' assessment}

\section{'Risk of bias' domains}

Random sequence generation (selection bias due to inadequate generation of a randomised sequence)

For each included trial, we will describe the method used to generate the allocation sequence in sufficient detail to allow an assessment of whether it should produce comparable groups

- Low risk of bias: the trial authors achieved sequence generation using computer-generated random numbers or a random numbers table. Drawing of lots, tossing a coin, shuffling cards or envelopes, and throwing dice are adequate if an independent person performed this who was not otherwise involved in the trial. We will consider the use of the minimisation technique as equivalent to being random.

- Unclear risk of bias: insufficient information about the sequence generation process.

- High risk of bias: the sequence generation method was non-random or quasi-random (e.g. sequence generated by odd or even date of birth; sequence generated by some rule based on date (or day) of admission; sequence generated by some rule based on hospital or clinic record number; allocation by judgment of the clinician; allocation by preference of the participant; allocation based on the results of a laboratory test or a series of tests; or allocation by availability of the intervention).

Allocation concealment (selection bias due to inadequate concealment of allocation prior to assignment)

We will describe for each included trial the method used to conceal allocation to interventions prior to assignment and we will assess whether intervention allocation could have been foreseen in advance of or during recruitment or changed after assignment

- Low risk of bias: central allocation (including telephone, interactive voice-recorder, internet-based and pharmacy-controlled randomisation); sequentially numbered drug containers of identical appearance; sequentially numbered, opaque, sealed envelopes.

- Unclear risk of bias: insufficient information about the allocation concealment.

- High risk of bias: used an open random allocation schedule (e.g. a list of random numbers); assignment envelopes used without appropriate safeguards; alternation or rotation; date of birth; case record number; any other explicitly unconcealed procedure.

We will also evaluate trial baseline data to incorporate assessment of baseline imbalance into the 'Risk of bias' judgment for selection bias (Corbett 2014). Chance imbalances may also affect judgments on the risk of attrition bias. In the case of unadjusted analyses, we will distinguish between trials that we rate as being at low risk of bias on the basis of both randomisation methods and baseline similarity, and trials that we judge as being at low risk of bias on the basis of baseline similarity alone (Corbett 2014). We will reclassify 
judgements of unclear, low or high risk of selection bias as specified in Appendix 3.

Blinding of participants and study personnel (performance bias due to knowledge of the allocated interventions by participants and personnel during the trial)

We will evaluate the risk of detection bias separately for each outcome (Hróbjartsson 2013). We will note whether endpoints were self-reported, investigator-assessed or adjudicated outcome measures (see below)

- Low risk of bias: blinding of participants and key study personnel was ensured, and it was unlikely that the blinding could have been broken; no blinding or incomplete blinding, but we judge that the outcome is unlikely to have been influenced by lack of blinding.

- Unclear risk of bias: insufficient information about the blinding of participants and study personnel; the trial does not address this outcome.

- High risk of bias: no blinding or incomplete blinding, and the outcome is likely to have been influenced by lack of blinding; blinding of trial participants and key personnel attempted, but likely that the blinding could have been broken, and the outcome is likely to be influenced by lack of blinding.

\section{Blinding of outcome assessment (detection bias due to knowledge of the allocated interventions by outcome assessment}

We will evaluate the risk of detection bias separately for each outcome (Hróbjartsson 2013). We will note whether endpoints were self-reported, investigator-assessed or adjudicated outcome measures (see below)

- Low risk of bias: blinding of outcome assessment is ensured, and it is unlikely that the blinding could have been broken; no blinding of outcome assessment, but we judge that the outcome measurement is unlikely to have been influenced by lack of blinding.

- Unclear risk of bias: insufficient information about the blinding of outcome assessors; the trial did not address this outcome.

- High risk of bias: no blinding of outcome assessment, and the outcome measurement was likely to have been influenced by lack of blinding; blinding of outcome assessment, but likely that the blinding could have been broken, and the outcome measurement was likely to be influenced by lack of blinding.

Incomplete outcome data (attrition bias due to amount, nature or handling of incomplete outcome data)

For each included trial or each outcome, or both, we will describe the completeness of data, including attrition and exclusions from the analyses. We will state whether the trial reported attrition and exclusions, and report the number of participants included in the analysis at each stage (compared with the number of randomised participants per intervention/comparator groups). We will also note if the trial reported the reasons for attrition or exclusion and whether missing data were balanced across groups or were related to outcomes. We will consider the implications of missing outcome data per outcome such as high dropout rates (e.g. above 15\%) or disparate attrition rates (e.g. difference of $10 \%$ or more between trial arms)

- Low risk of bias: no missing outcome data; reasons for missing outcome data unlikely to be related to true outcome (for survival data, censoring unlikely to introduce bias); missing outcome data balanced in numbers across intervention groups, with similar reasons for missing data across groups; for dichotomous outcome data, the proportion of missing outcomes compared with observed event risk was not enough to have a clinically relevant impact on the intervention effect estimate; for continuous outcome data, plausible effect size (mean difference or standardised mean difference) among missing outcomes was not enough to have a clinically relevant impact on observed effect size; appropriate methods, such as multiple imputation, were used to handle missing data.

- Unclear risk of bias: insufficient information to assess whether missing data in combination with the method used to handle missing data were likely to induce bias; the trial did not address this outcome.

- High risk of bias: reason for missing outcome data was likely to be related to true outcome, with either imbalance in numbers or reasons for missing data across intervention groups; for dichotomous outcome data, the proportion of missing outcomes compared with observed event risk enough to induce clinically relevant bias in the intervention effect estimate; for continuous outcome data, plausible effect size (mean difference or standardised mean difference) among missing outcomes enough to induce clinically-relevant bias in observed effect size; 'as-treated' or similar analysis done with substantial departure of the intervention received from that assigned at randomisation; potentially inappropriate application of simple imputation.

\section{Selective reporting (reporting bias due to selective outcome reporting)}

We will assess outcome reporting bias by integrating the results of the appendix 'Matrix of trial endpoints (publications and trial documents)' (Boutron 2014; Jones 2015; Mathieu 2009), with those of the appendix 'High risk of outcome reporting bias according to the Outcome Reporting Bias In Trials (ORBIT) classification' (Kirkham 2010). This analysis will form the basis for the judgement of selective reporting

- Low risk of bias: the trial protocol was available and all the trial's prespecified (primary and secondary) outcomes that were of

Parathyroidectomy for adults with primary hyperparathyroidism (Protocol) 


\section{(Continued)}

interest to this review were reported in the prespecified way; the study protocol was unavailable, but it was clear that the published reports included all expected outcomes (ORBIT classification).

- Unclear risk of bias: insufficient information about selective reporting.

- High risk of bias: not all the trial's prespecified primary outcomes were reported; one or more primary outcomes were reported using measurements, analysis methods or subsets of the data (e.g. subscales) that were not prespecified; one or more reported primary outcomes were not prespecified (unless clear justification for their reporting was provided, such as an unexpected adverse effect); one or more outcomes of interest in the Cochrane Review were reported incompletely so that we cannot enter them in a meta-analysis; the trial report failed to include results for a key outcome that we would expect to have been reported for such a trial (ORBIT classification).

\section{Other bias}

- Low risk of bias: the trial appears to be free from other sources of bias.

- Unclear risk of bias: there was insufficient information to assess whether an important risk of bias existed; insufficient rationale or evidence that an identified problem introduced bias.

- High risk of bias: the trial had a potential source of bias related to the specific trial design used; the trial was claimed to be fraudulent; or the trial had some other serious problem.

\section{Appendix 3. Selection bias decisions}

Selection bias decisions for trials that reported unadjusted analyses: comparison of results obtained using method details alone with results using method details and trial baseline information ${ }^{a}$

\begin{tabular}{l|l|l|l|l|l|l|l|l}
\hline $\begin{array}{l}\text { Reported randomisation and } \\
\text { allocation concealment meth- } \\
\text { ods }\end{array}$ & $\begin{array}{l}\text { Risk of bias judgement using } \\
\text { methods reporting }\end{array}$ & $\begin{array}{l}\text { Information gained from } \\
\text { study characteristics data }\end{array}$ & $\begin{array}{l}\text { Risk of bias using baseline } \\
\text { information and methods re- } \\
\text { porting }\end{array}$ \\
\hline
\end{tabular}

Unclear methods $\quad$ Unclear risk

\section{Baseline imbalances present for High risk} important prognostic variable

(s)
Groups appear similar at base- Low risk line for all important prognos- tic variables

Limited or no baseline details Unclear risk

Would generate a truly random Low risk sample, with robust allocation concealment
Baseline imbalances present for Unclear risk $^{b}$ important prognostic variable

(s)

Groups appear similar at base- Low risk line for all important prognostic variables

Limited baseline details, show- Low risk ing balance in some important prognostic variables ${ }^{c}$ 


\begin{tabular}{l|l}
\cline { 2 - 3 } $\begin{array}{l}\text { Sequence is not truly ran- } \\
\text { domised or allocation conceal- } \\
\text { ment is inadequate }\end{array}$ & $\begin{array}{l}\text { No baseline details } \\
\text { Baseline imbalances present for } \\
\text { important prognostic variable } \\
(\mathrm{s})\end{array}$ \\
\cline { 2 - 3 } & $\begin{array}{l}\text { Groups appear similar at base- } \\
\text { line for all important prognos- } \\
\text { tic variables }\end{array}$ \\
\hline & $\begin{array}{l}\text { Limited baseline details, show- } \\
\text { ing balance in some important } \\
\text { prognostic variables }\end{array}$ \\
\hline & No baseline details risk \\
\hline
\end{tabular}

${ }^{a}$ Taken from Corbett 2014; judgements highlighted in bold indicate situations in which the addition of baseline assessments would change the judgement about risk of selection bias, compared with using methods reporting alone.

${ }^{b}$ Imbalance identified that appears likely to be due to chance.

${ }^{c}$ Details for the remaining important prognostic variables are not reported

\section{CONTRIBUTIONSOFAUTHORS}

All review authors contributed to, read and approved the final protocol draft.

\section{DECLARATIONSOF INTEREST}

Joseph M Pappachan (JMP): none known.

Ravinder Sodi (RS): none known.

Ananth K Viswanath (AKV): received speakers fees for providing diabetes educational meetings for GPS/Primary care colleagues. These events were supported by various pharmaceutical companies. Similarly received support from pharmaceutical companies to attend international diabetes conferences.

Ian M Lahart (IML): none known. 


\section{NOTES}

We have based parts of the Methods, as well as Appendix 1, Appendix 2 and Appendix 3 of this Cochrane Protocol on a standard template established by the CMED group. 